www.jmscr.igmpublication.org

Index Copernicus Value: 79.54

ISSN (e)-2347-176x ISSN (p) 2455-0450

crossref DOI: https://dx.doi.org/10.18535/jmscr/v7i6.52

\title{
Diagnostic Role of RDW \& PDW as Early Markers of Sepsis in Chemotherapy Induced Febrile Neutropenia
}

\author{
Authors \\ Nitin Mohan ${ }^{1}$, Veni Prasanna Gedela ${ }^{2}$, Murali Krishna Voona ${ }^{3}$, Viswa Kalyan Kolli ${ }^{*}$ \\ ${ }^{1}$ Department of Microbiology, GITAM Institute of Medical Sciences \& Research, GITAM (Deemed to be \\ University), Rushikonda, Visakhapatnam - 530045, Andhra Pradesh, India \\ ${ }^{2}$ Hemato-Pathologist, Mahatma Gandhi Cancer Hospital \& Research Institute, 1/7, MVP Colony, \\ Visakhapatnam - 530017.Andhra Pradesh, India \\ ${ }^{3}$ Surgical Oncologist, Mahatma Gandhi Cancer Hospital \& Research Institute, 1/7, MVP Colony, \\ Visakhapatnam - 530017.Andhra Pradesh, India
}

${ }^{4}$ Department of Biochemistry, GITAM Institute of Medical Sciences \& Research, GITAM (Deemed to be

University), Rushikonda, Visakhapatnam - 530045, Andhra Pradesh, India

*Corresponding Author

Dr Viswa Kalyan Kolli

Associate Professor, Department of Biochemistry, GITAM Institute of Medical Sciences \& Research,

GITAM (Deemed to be University), Rushikonda, Visakhapatnam - 530045. Andhra Pradesh. India

\begin{abstract}
Introduction: Febrile neutropenia is commonly seen in patients undergoing chemotherapy that makes them susceptible to infections. Procalcitonin (PCT) is routinely used as a marker of systemic infections. Recently, Red Cell Distribution Width (RDW) and Platelet Distribution Width (PDW) were also indicated for the presence of infections. The aim of the study was to find the clinical utility of RDW and PDW along with PCT as biomarker for early institution of antibiotic therapy.

Materials \& Methods: 150 patients with haematological malignancies who had chemotherapy-induced neutropenia were included in the study and were tested for PCT, RDW and PDW.

Results: 42 patients who tested negative for PCT were considered as Control and the 108 patients who tested positive for PCT were considered as Test. RDW and PDW values were significantly increased in the test group (RDW: $51.9+10.29$ and PDW: 12.2+3.56)as compared to the control group (RDW (SD): $43.49+7.98$ and $P D W: 10.76+1.78)$.

Conclusion: RDW and PDW together with PCT can be used as biomarker for sepsis and early institution of antibacterial therapy.

Keywords: Procalcitonin (PCT); Red Cell Distribution Width (RDW); Platelet Distribution Width (PDW); Sepsis.
\end{abstract}

\section{Introduction}

Febrile Neutropenia is commonly seen in cancer patients undergoing chemotherapy. Infections can occur with minimal signs and symptoms and progress rapidly. In such cases the major challenge is whether to start antibiotic therapy or not. Blood culture is the classical and gold standard approach for identification of sepsis. 
However, it takes 2-5 days for the blood culture reports to be available; other markers for sepsis are being explored so that the decision on the institution of antibiotic therapy can be taken early. Among the known markers, Procalcitonin (PCT) and C-Reactive Protein (CRP) have become an important tool for early diagnosis of systemic infections. PCT is a prohormone of calcitonin (CT) produced mainly by $\mathrm{C}$-cells of the thyroid gland with a molecular weight of $13 \mathrm{kDa}$. In a healthy condition or in the absence of infections, its blood level is very low $(<0.1 \mathrm{ng} / \mathrm{mL})$, but in case of infection, its level is known to increase rapidly ${ }^{[1]}$.

The red blood cell distribution width (RDW) and platelet distribution width (PDW) are parts of a routine complete blood count. RDW is a means of evaluating the variability in size of erythrocytes and has been used widely in the differential diagnosis of anemia ${ }^{[2]}$. PDW is the measure of variation in platelet size, which may be an indicator of active platelet release ${ }^{[3]}$. Recently, RDW and PDW were found to significantly associate with chronic spontaneous urticaria and gram negative bacteremia ${ }^{[4][5]}$.

The aim of the study is to find the clinical utility of RDW and PDW along with PCT as biomarker for early institution of antibiotic therapy.

\section{Materials \& Methods}

The study was conducted in a tertiary care cancer hospital and is a prospective observational study.

\section{Study Population}

Patients with haematological malignancies who had chemotherapy-induced neutropenia $(<1,500$ neutrophils per microliter of blood) and tested for PCT were included in the study. 150 patients who have fulfilled the above criteria were included in the study.

\section{Sample Collection}

After taking informed consent, $5 \mathrm{~mL}$ of venous blood was collected from the patients under aseptic conditions.

\section{Estimation of Procalcitonin}

Procalcitonin was measured by Thermo Scientific $\mathrm{B} \cdot \mathrm{R} \cdot \mathrm{A} \cdot \mathrm{H} \cdot \mathrm{M} \cdot \mathrm{S}$ PCT-Q, an immunochromatographic point-of-care test. $200 \mu \mathrm{L}$ of serum was pipetted into the round cavity and the time was documented on the reference card. After 30 minutes of incubation at room temperature, the concentration range of the sample was determined by comparing the color intensity of the test band with the colored blocks of the reference card. Bands corresponding to $>0.5,>2.0$ and $>10.0$ indicate systemic infection.

\section{Estimation of RDW and PDW}

The blood counts were measured using the Sysmex-XN 350 analyzer. The Mean \pm SD and $\% \mathrm{CV}$ values of quality control samples for RDW and PDW are RDW(SD): $51.3 \pm 0.33$ (0.6); PDW: $8.1 \pm 0.19$ (2.4).

\section{Study Groups}

Control Group: 42 patients with PCT $<0.5$ served as Control group.

Test group: 108 patients with PCT $>0.5$ served as Test group.

\section{Statistical Analysis}

Statistical analysis was done with SPSS software. Student t-test was used to find the difference between the groups. P Value of $<0.05$ was considered significant.

\section{Results}

Out of the 150 patients, 42 patients showed no PCT band $(<0.5)$ and 108 patients showed PCT band indicating systemic infection. Out of 108, 42 have shown band intensity corresponding to $0.5,40$ have shown band intensity corresponding to $2.0,26$ have shown band intensity corresponding to 10.0. (Table. 1).

In the control group, the Mean+SD values are RDW (SD): $43.49 \pm 7.98$ and PDW: $10.76 \pm 1.78$.In the test group, the Mean \pm SD values of RDW (SD): $51.9 \pm 10.29$ and PDW: 12.2 \pm 3.56 were significantly higher as compared to control. (Table. 2). 
Table 1: \% distribution of PCT positive cases among the test group

\begin{tabular}{|l|c|c|}
\hline PCT Values & No. of cases & $\%$ \\
\hline 0.5 & 42 & 39 \\
\hline 2 & 40 & 37 \\
\hline 10 & 26 & 24 \\
\hline Total & 108 & \\
\hline
\end{tabular}

Table 2: Mean \pm SD values of RDW (SD) and PDW in the control and test groups.

\begin{tabular}{|l|c|c|c|c|}
\hline Variables & Groups & Mean & SD & P-value \\
\hline \multirow{2}{*}{ RDW (SD) } & Control & 43.49 & 7.98 & \multirow{2}{*}{$<0.001$} \\
\cline { 2 - 4 } & Test & 51.92 & 10.29 & \\
\hline \multirow{2}{*}{ PDW } & Control & 10.76 & 1.78 & \multirow{2}{*}{$<0.001$} \\
\cline { 2 - 4 } & Test & 12.17 & 3.56 & \\
\hline
\end{tabular}

\section{Discussion}

Patients undergoing chemotherapy for hematological malignancies are more prone to neutropenia - a state of abnormal low number of neutrophils and are more prone to infections. Blood culture remains the gold standard method to confirm systemic infection. Recently, procalcitonin is being used as marker of infection. This will reduce the reporting time for the blood culture samples and helps in the decision making for early administration of antibiotic therapy. Since recent studies on $\mathrm{RDW}^{[6][7]}$ and $\mathrm{PDW}^{[8]-[10]}$ have shown its positive correlation with inflammation and infectious diseases, the current study was carried out to find the clinical utility of RDW and PDW along with procalcitonin for the early institution of antibiotic therapy in neutropenic hematological malignancy patients.

Earlier studies on procalcitonin levels have shown its significant correlation with sepsis ${ }^{[11]}$. PCT is being used as a biomarker for antibiotic therapy in the management of patients with infection/ sepsis across various clinical settings ${ }^{[12]-[14]}$.

Based on the above studies, we have chosen PCT as a marker of infection in febrile neutropenic patients and observed the changes in RDW and PDW values. The results showed that RDW and PDW values were significantly higher in the test group. Zhang $\mathrm{HB}$ et al. have shown that a combination of the three parameters (RDW, PDW
\& Neutrophil-lymphocyte count ratio) showed similar diagnostic precision to that of PCT in nonhematological malignancy patients ${ }^{[15]}$. Thus, we conclude that routine blood count parameters RDW and PDW values can be used as new biomarker along with PCT for the early institution of antibacterial therapy in neutropenic patients with hematological malignancies.

\section{Conclusion}

RDW and PDW together with PCT can be used as biomarker for sepsis and early institution of antibacterial therapy.

\section{References}

1. Liu HH, Guo JB, Geng Y, Su L. Procalcitonin: present and future. Ir J Med Sci.2015;184(3): 597-605.

2. Miyamoto $\mathrm{K}$, Inai $\mathrm{K}$, Takeuchi $\mathrm{D}$, Shinohara $\mathrm{T}$ and Nakanishi $\mathrm{T}$. Relationships among red cell distribution width, anemia and interleukin- 6 in adult congenital heart disease. Circ J. 2015;79(5):1100-1106.

3. Kasperska-Zajac A, Grzanka A, Jarzab J, Misiołek M, Wyszyńska-Chłap M, Kasperski J, Machura E. The association between platelet count and acute phase response in chronic spontaneous urticaria. Biomed Res Int. 2014;2014:650913.

4. Ku NS, Kim HW, Oh HJ, Kim YC, Kim $\mathrm{MH}$, Song JE, et al. Red blood cell distribution width is an independent predictor of mortality in patients with gram-negative bacteremia. Shock. 2012;38(2):123-127.

5. Celik A, Aydin N, Ozcirpici B, Saricicek E, Sezen H, Okumus M, et al. Elevated red blood cell distribution width and inflammation in printing workers. Med Sci Monit. 2013;19:1001-1005.

6. Lippi G, Targher G, Montagnana M, Salvaqno GL, Zoppini G, Guidi GC. Relation between red blood cell distribution width and inflammatory 
biomarkers in a large cohort of unselected outpatients. Arch Pathol Lab Med. 2009;133(4):628-632.

7. Kucukbayrak A, Tas T, Tosun M, Aktas G, Hakyemez IN, Mengeloglu FZ. Erythrocytes parameters in the course of Brucellosis. Abant Med J. 2013;2(1):3638.

8. Ahmad MS, Waheed A. Platelet counts, MPV and PDW in culture proven and probable neonatal sepsis and association of platelet counts with mortality rate. J Coll Physicians Surg Pak. 2014;24(5):340-344.

9. Abdel-Razik A, Eldars W, Rizk E. Platelet indices and inflammatory markers as diagnostic predictors for ascitic fluid infection. Eur J Gastroenterol Hepatol. 2014;26(12):1342-1347.

10. Orak M, Karakoç Y, Ustundag M, Yildirim Y, Celen MK, Güloglu C. An investigation of the effects of the mean platelet volume, platelet distribution width, platelet/lymphocyte ratio, and platelet counts on mortality in patents with sepsis who applied to the emergency department. Niger J ClinPract. 2018;21(5):667-671.

11. Rebello A, Thabah MM, Dutta TK, Bobby Z, Harish BN, Mehalingam V. Procalcitonin levels in sepsis and its association with clinical outcome in southern India. Trop Doct. 2017;47(4):331-336.

12. Schuetz P, Christ-Crain M, Thomann R, Falconnier C, Wolbers M, Widmer I, et al. Effect of procalcitonin-based guidelines vs standard guidelines on antibiotic use in lower respiratory tract infections: the ProHOSP randomized controlled trial. JAMA. 2009; 302(10): 1059-1066.

13. Bouadma L, Luyt CE, Tubach F, Cracco C, Alvarez A, Schwebel C, Schortgen F, et al. Use of procalcitonin to reduce patients' exposure to antibiotics in intensive care units

(PRORATA

trial):

a

multicentrerandomised controlled trial. Lancet. 2010;375(9713):463-474.

14. Schuetz P, Bretscher C, Bernasconi L, Mueller B. Overview of procalcitonin assays and procalcitonin-guided protocols for the management of patients with infections and sepsis. Expert Rev MolDiagn. 2017;17(6):593-601.

15. Zhang HB, Chen J, Lan QF, Ma XJ, Zhang SY. Diagnostic values of red cell distribution width, platelet distribution width and neutrophil-lymphocyte count ratio for sepsis. ExpT her Med. 2016;12(4):2215-2219. 